\title{
Incommensurability, types of phenomena and relevant incompatibility (part III)
}

Esteban Céspedes (estebancespedes@aol.com) Instituto de Sistemas Complejos de Valparaíso (Valparaíso, Chile) ORCID: 0000-0002-5329-5434

\begin{abstract}
This is the final part of a three-part paper. In the first part, it was proposed that a notion of phenomenon type could be relevant to tackle some crucial issues about incommensurability. Then, part II had a focus on some remaining problems associated with scientific realism and the possibility of scientific progress. In this part, some ways to solve them are offered, without abandoning the classical features of incommensurability. This can be done by showing that two rival theories may refer to the same phenomenon type even if their ontologies are incompatible and assuming at the same time a non-naïve, moderate scientific realism.
\end{abstract}

Key words: incommensurability, sense, reference, phenomenon type, scientific realism.

\section{Scientific progress based on a coherence theory of reality}

Following the notion of a subject matter as presented in the previous section (part II), suppose that differing properties $M_{T}$ and $M_{T^{*}}$ relate to a subject matter $\Phi$. On this basis, we can compare the incommensurable theories $T$ and $T^{*}$, as well as the corresponding extensionally differing terms $m_{T}$ and $m_{T^{*}}$. We can achieve this comparison making sense of scientific progress. Consider the following formulation:

(28) Scientific progress. Any theoretical change within the investigation of a subject matter may involve a better understanding of that matter.

Note that (28) is compatible with realist scientific progress (see characterisation 21). The crucial question is whether the set of entities investigated by theories are independent from theoretical descriptions. I think that we do not have to consider them that way. Furthermore, we may clarify the difficulties surrounding the possibility of incommensurability by accepting that scientific theories are not mainly concerned with descriptions of an ultimate reality.

Regarding the type of phenomenon associated with mass as a subject matter, we may claim that, even if the Newtonian concept of mass is prima facie incompatible with the relativistic concept of mass, the possibility of carrying out a theoretical change between Newtonian mechanics and special relativity implies a better understanding of that type of phenomenon.

Figure 2 represents the roles that phenomena, scientific terms and their referents play in the development of scientific inquiry. 
Experiences of type $\varepsilon_{1}$ conform to predictions
Experiences of types $\varepsilon_{1}$ and

$\varepsilon_{2}$ conform to predictions

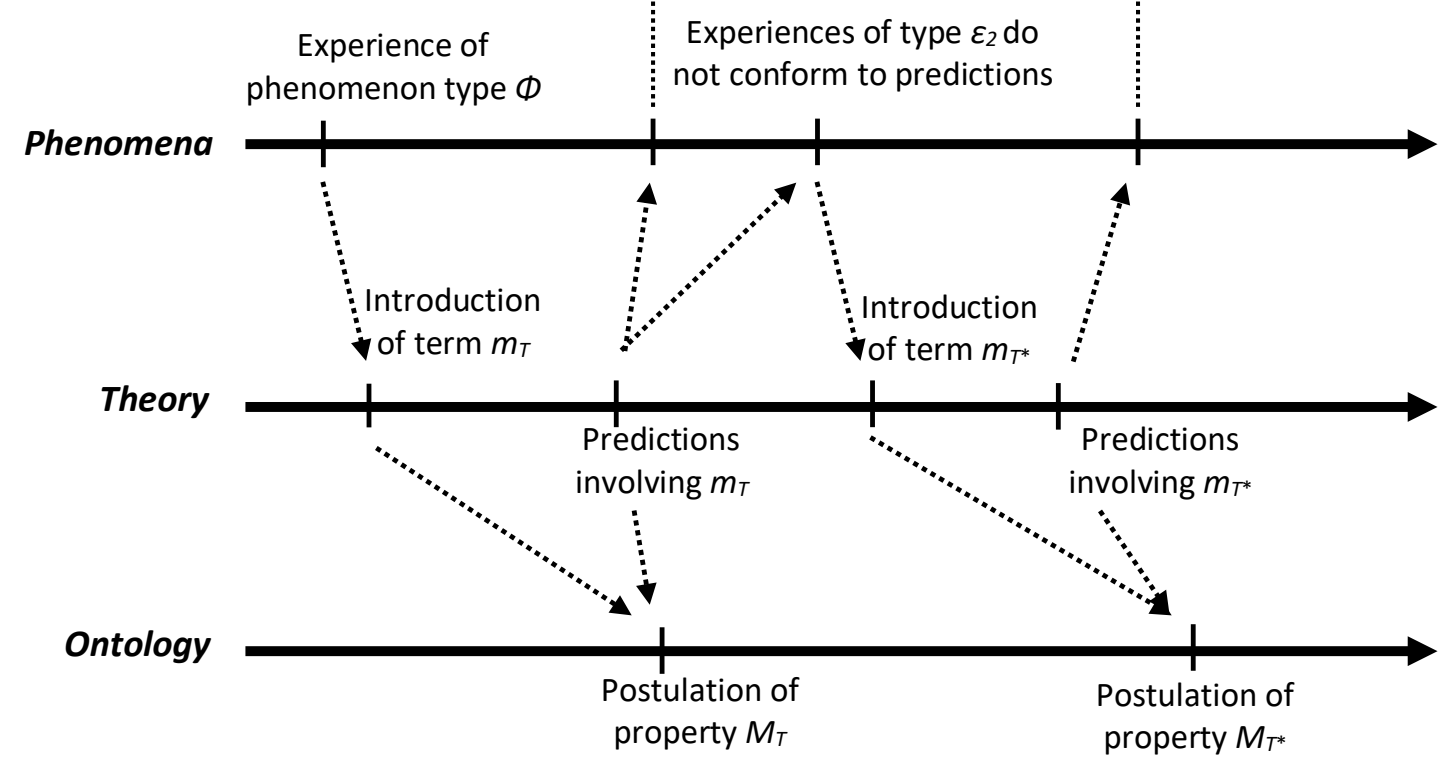

Fig. 2. Theoretical and ontological development

Experiences, i.e. phenomena, of types $\varepsilon_{1}$ and $\varepsilon_{2}$ can be considered as special types of the more general phenomenon type $\Phi$. Although the terms $m_{T}$ and $m_{T^{*}}$, as well as the ontologies associated with them, may be incompatible, the theories in which they are introduced are based, considering their development, on the same general phenomenon type.

Note also that the ontology associated with a term, i.e. its theoretical reference, varies with theory. This is the key point of the description theory of reference. The ontology is not absolute but consists of a set of assumptions based on the commitments imposed by a theory.

Another important point to consider is how the theory is based on the phenomena. These also determine the subject matter of scientific inquiry, which, of course, may also vary. Thus, the picture proposed here involves a non-naïve realist sense of scientific progress. One main thesis concerning realism is the following: First, our understanding of the ontology is improved thanks to theoretical improvement. Scientific theories are thus actually focused on a set of entities. However, it is not an absolute and independent set, but a set that is determined by changing subject matters, types of phenomena and theoretical description. I do not see any reason to claim that this perspective cannot be a kind of realism. The realist aspect of it does not lie on the side of an independent reality, but on the side of the ontology. This proposal is in the line of Hasok Chang's pragmatic realism (Operational coherence as the source of truth), as opposed to naïve realism. He defends a coherence theory of reality, which is based on the following main thesis:

(29) Coherence theory of reality. Entities can be considered as real if they are part of a coherent epistemic activity that relies on their existence and its basic properties. 
Note that the notion of coherence involved in this thesis does not refer to mere logical coherence, but to pragmatic coherence. Following Chang's view, we may claim that any epistemic activity is crucially constituted by phenomenon types. What we consider as part of our ontology (i.e. as real) depends on them, as well as on other factors, of course. Theoretical improvement, together with our ontological commitments, improves our understanding of the phenomena. In this sense, the postulated ontologies are not just products of theories, but they also support novel, scientific development.

It is usually argued that the possibility of incommensurability implies that scientific development does not consist in an increase of verisimilitude or an approximation to a true picture of nature, that is, truth. I agree that it is perhaps impossible to determine, comparing two incommensurable theories, which of the two postulated ontologies is closer to the absolute and purely objective ontology (whatever that may be). However, it is possible to determine which theory explains better the phenomena. If the true picture of nature is characterised as the best possible way according to which the available set of phenomena could be explained, then we can claim that, in this sense, scientific development consists in an approximation to truth.

John Collier (Pragmatic incommensurability) also proposes to account for incommensurability based on pragmatic aspects. According to his proposal, rival theories can be compared based on interpretation techniques, which are constitutive parts of theories that provide common means to grasp the relevant concepts grounding the conflict. This point is interestingly compatible with the proposal based on phenomenon types, since it considers the importance of non-extensional aspects involved in conceptual understanding.

In a similar way, Hoyningen-Huene proposes to characterise incommensurability based on the Kantian notion of phenomenon. According to him, scientific knowledge must be conceived as knowledge about phenomena. However, the present proposal differs from his in some relevant aspects. Hoyningen-Huene claims that "incommensurable theories approach reality from different phenomenal worlds and that they target, nevertheless, the same domain of the world-in-itself" (1993:219). Now, according to the notion of incommensurability considered in this work, the condition that makes possible the comparison between incommensurable theories is precisely the fact that they target a common domain of phenomena. This point of comparison is constituted by types of phenomena, not by a particular phenomena. Sure, incommensurable theories involve different phenomenal worlds, regarding both types of phenomena. But they also share a domain of relevant phenomenon types, which is what allows us to compare them. Furthermore, by taking phenomenon types as points of comparison, we do not have to speculate about the world-in-itself, being thus faithful to Kant's epistemology.

Sankey (Scientific realism and the semantic incommensurability thesis) criticises the Kantian distinction between phenomena and noumena and, particularly, accounts like the one defended by HoyningenHuene and Feyerabend (Problems of empiricism) for trying to say too much about the world-in-itself. The perspective proposed here does not fall in the latter problem, although it implies the mentioned distinction indeed: We can talk, in a transcendental sense, about an independent world as opposed to the domain of phenomena and as the transcendent cause of any singular phenomenon. But it would be incoherent with Kant's theory to say that some scientific terms of rival theories can refer to the same domains of the world-in-itself, as Feyerabend and Hoyningen-Huene do. 
Following these ideas, which are perfectly compatible with a characterisation of semantic incommensurability, realism about scientific progress is not threatened. Only the notion of an invariable and absolute ontology is.

The point of view proposed here is coherent with Bas van Fraassen's constructive empiricism (The scientific image), a perspective according to which the acceptance of a scientific theory depends only on its empirical adequacy, that is, on whether it "saves the phenomena". In van Fraassen's account, a theory is empirically adequate if its descriptions about observable things and events are true. I prefer here to be careful with the notion of truth. However, at the end of this article I consider a notion of prediction that may be compatible, under certain assumptions, with the notion of empirical adequacy involved in constructive empiricism. Such a notion of empirical adequacy is a structuralist notion of adequacy: Descriptions are adequate if their structure fits the structure of the corresponding phenomena. Now, van Fraassen's defends a non-realist version of structuralism, arguing that one may remain agnostic about the reality of some entities. I agree, one may do that. But it is more rational sometimes, for the sake of scientific progress, to postulate corresponding ontologies, even in cases of incommensurability and even if those ontologies result to be incompatible.

\section{Realism about incommensurability}

How should we consider the problem that is apparently faced by realism about scientific progress because of accepting the possibility of incommensurability? Roughly, I would say that incommensurability is as real as the properties that scientific theories try to describe. As Howard Sankey (Scientific realism and the semantic incommensurability thesis) considered the problem discussed above, it seems that we lose realism if we accept that incommensurability is possible. Thus, the solution might be to accept realism and reject the possibility of incommensurability. But that is not the only alternative. The problem of incommensurability is an epistemic issue, just as the question of whether the properties introduced by scientific theories have an independent, objective reality. On this basis, I do not consider the incommensurability relation between two theories as a relation that holds objectively and independently of the considerations about the development of scientific knowledge to which one may point.

As I argued above, the meaning variance in cases of incommensurability may involve incompatibility of sense as well as of reference. One may claim, on this basis and based on a Kantian point of view, that the incommensurability thesis threatens naïve realism because it is not possible that theories refer to an independent reality understood as a world-in-itself (Hoyningen-Huene; Oberheim; Andersen On incommensurability). According to the account of incommensurability presented here, the notion of a world-in-itself is not considered, but it could be characterised as the metaphysical ground on which the sets of data, i.e. the phenomena, are based. Anyhow, such a notion is neither needed in order to understand theoretical incommensurability nor to formulate a notion of scientific progress. In this sense: "claims about the world-in-itself should not be understood as claims regarding our acquired and always improving knowledge about a purely objective reality, but as philosophical postulates that help us to understand knowledge, grounded on inter-subjective projections of several phenomenal worlds" (Hoyningen-Huene \& Oberheim 2009:207), i.e. they must not be viewed from the perspective of naïve realism, which may be characterised as follows:

(30) Naïve realism. The reference of a term $t$ is an entity that exists independently from the language in which $t$ was introduced and is the basis of every phenomenon. 
Here, the reference of a term is understood as a thing-in-itself. Thus, the main difference between naïve realism and the point of view defended here is based on this: According to naive realism, one can say something meaningful about the thing-in-itself besides the fact that it grounds the phenomena. By contrast, according to the notion of ontology implied by the present proposal on incommensurability, one cannot.

Incommensurability is better understood under the assumption that the reference of a theoretical property is not simply a set of objects that exist independently of theory, but is a feature determined by the formal descriptions and the set of possible predictions that are available on the basis of the theory in which the term associated with it is defined. Thus, considering that incommensurability seems paradoxical only within a naïve realist point of view and that there is an important historical support for incommensurability, we have good reasons to reject naïve scientific realism.

\section{Agreement domains of prediction based on common phenomenal reference}

According to Paul Hoyningen-Huene and Eric Oberheim (Reference, ontological replacement and neokantianism), one should distinguish between the entities to which a theory refers on the basis of a certain term $t$ and the entities to which a prediction may refer on the basis of $t$ within the framework of that theory. We may understand this distinction in a clearer way by considering the following pair of claims:

(31) The term $t$ involved in the formulation of theory $T$ refers to a certain property $P$.

(32) The term $t$ involved in a prediction of $T$ refers to an instantiation of the property $P$.

While the formulation of a theory refers to general properties, predictions refer to cases. For example, the Newtonian term "mass" refers, according to the formulation of the second law of motion, to a certain property. One can assume that such a property was extracted through generalisations from the set of phenomena based on which theory was formulated. Now, when one predicts the mass of an object within the framework of Newtonian mechanics, the term "mass" involved in the prediction refers to a property instantiated in that particular (possible) event.

Let $T$ and $T^{*}$ be two incommensurable theories. Now suppose the following:

(33) Within a domain of prediction, the term $t$ involved in the predictions generated by theory $T$ and the term $t^{*}$ involved in the predictions generated by theory $T^{*}$ refer to the same entities.

This supposition does not imply the following claim (Hoyningen-Huene \& Oberheim. Reference, ontological replacement and neo-Kantianism):

(34) The term $t$ involved in the formulation of $T$ and the term $t^{*}$ involved in the formulation of $T^{*}$ refer to the same entities.

Thus, in the case of two mutually incommensurable theories, an instance of (33) may be true while the suited instance of (34), involving the same theoretical terms, may be false. This is the sort of incompatibility that is usually considered regarding the term "mass" when Newtonian mechanics is compared to special relativity. Regarding a certain domain, Newtonian mechanics achieves the same predictions as relativity theory and thus, the term "mass" refers to the same entities in those cases. Considering such a restriction, 
the term "mass" that occurs in the set of predictions formulated within Newtonian mechanics has the same reference, we may assume, as the term "mass" that occurs in the set of predictions formulated within relativity theory. Nevertheless, given that both theories are incommensurable regarding the phenomenon type related to mass, the term "mass" that occurs in the general formulation of Newtonian mechanics has not the same reference as the term "mass" that occurs in the main equation of relativity theory.

Note that this way of considering the incommensurability between scientific theories does not imply that theoretical reference is a relation between language and a purely independent world. Both Feyerabend and Hoyningen-Huene somehow support this view. Rather, theoretical reference can be seen a relation between language and a postulated ontology. The assumed purely independent world should be understood as a transcendental anchorage point that sustains the phenomena. Of course, a phenomenon has already a certain structure grounded on a set of basic properties, but, since these are qualitative properties, it would not be necessary to associate them with a previously given, independent reality. These may be determined by a previous conceptual apparatus or metalanguage. For instance, as already mentioned, one may claim that the phenomenon type on which the Newtonian concept of mass is based is the apparent resistance of material bodies to change their motion state. Such a phenomenon type involves some basic features due to a conceptual structure that is distinct from the Newtonian theory. Thus, Newtonian mechanics introduces the theoretical term of mass in order to account for the regularities that may be extracted from the initial set of phenomena.

The main reason of the difference between (31) and (32) may be understood as follows. While predictions are claims pointing to phenomena, laws are obtained from generalisations of phenomena. One may suppose that the instance of a predicate, as it is considered in a prediction, refers to some particular entity and that a general term, as it is formulated in a law, refers to a property. Thus, the prediction that a object $a$ will instantiate the property $M$ at a given time expresses that it would be possible to observe a certain phenomenon at that moment, which could be described by saying that $a$ instantiates the property $M$. According to that phenomenon, the term associated with the instantiation of $M$, i.e. the term "mass", tells us something about the possible aspect of the entity that may produce that phenomenon. (However, it tells us that only in a speculative way, so that we are not actually describing the world-in-itself, which is impossible.) Now, when the term "mass" appears in the formulation of a law, it refers to a general entity, a general property of mass.

It should be noticed that although the reference of a term as it appears in a general formulation differs from the reference of the same term if it appears in a description or a prediction of a phenomenon, they are not fully independent from each other. Following the notion of theoretical reference defended here, the theory imposes an ontology of general properties that also allows to assume that there are instances of such properties. Suppose the following:

(35) On the basis of the term "mass", which is part of the formulation of a theory $T$, we may introduce the property $M$ into the ontology.

Let us now apply this to a description of a observed phenomenon $\varphi$ :

(36) Let $p$ be a description or a prediction, formulated within a theory $T$, of the observation of a phenomenon. If $p$ involves the term "mass", we may assume that such a term refers to the instantiation of property $M$ by a certain object $a$ at some time. 
Now, in which exact sense can we say that, within a predictive domain, the term "mass" involved in predictions of Newtonian mechanics may have the same reference as the term "mass" involved in predictions of special relativity? In order to answer this question, one must focus on the observation of the phenomenon involved. Consider the following idea of common reference:

(37) Common phenomenal reference under a given domain of prediction. A term $m_{T 1}$ involved in a prediction $p_{T 1}$, within a theory $T_{1}$, has the same reference as the term $m_{T 2}$ involved in a prediction $p_{T 2}$, within a theory $T_{2}$, just in case a suited observation mechanism observing the phenomenon predicted by $p_{T 1}$ would have produced essentially the same phenomenon if its observation had been based on prediction $p_{T 2}$.

We may further assume that phenomenal reference be a special sort of theoretical reference: If two agents experienced the same phenomenon, following a theory, they should make the same basic ontological suppositions regarding the entities to which their experiences supposedly correspond.

Also, the idea of common phenomenal reference expresses clearly the fact that, for instance, within a determined domain, the predictions of Newtonian mechanics and special relativity are equivalent. Outside such a domain, say, considering a domain in which extremely high velocities are involved, the phenomenon experienced by an observer that based her prediction on the Newtonian term of mass would differ from the phenomenon that she would have observed, if she had based her procedure on the relativistic term of mass. (Note, however, that this holds for traditional formulations of both theories. More recent formulations allow for closer connections between them, extending the Newtonian framework to be applied under conditions of extreme speeds. These cases are beyond the scope of the present article, but not beyond the interpretation of my proposal.)

These ideas are in line with Feyerabend's pragmatic theory of perception (An attempt at a realistic interpretation of experience; Problems of empiricism). He claimed that perceptions are indicators and, as such, they need to be interpreted. Considering the terminology proposed here, we may say that phenomena are indicators, but only if they are taken in their non-private aspects, i.e. only as phenomenon types. As shown in the present work, phenomenon types guide theoretical descriptions and, in line with Feyerabend's ideas, they need to be interpreted and described.

There is a problem with the explanation just given about the difference between a term being involved in predictions and in laws. Let us remember that singular phenomena are, per definition, private. How could we be able to formulate, communicate, contrast and validate predictions about phenomena if they cannot be shared? According to (37), the common phenomenal reference of distinct predictions is only counterfactual. Thus, the formulation, communication, comparison and validation of prediction would be also merely counterfactual. Considering this, we may differ in a certain way with Hoyningen-Huene and Oberheim (Reference, ontological replacement and neo-Kantianism), claiming, on the one hand, that we cannot understand some terms involved in predictions as referring to particular cases, at least concerning intensional reference. These predictions refer, in this sense, to phenomenon types. On the other hand, regarding the extensional reference of scientific terms, there does not seem to be a relevant difference between both accounts. The term mass, e.g., as it appears in a prediction, can extensionally refer both to general properties and to singular instantiations of properties in the ontology. This also holds for the term mass as it appears in a general formulation or a law. 
Now, what is then the difference between a term appearing in a prediction and the same term appearing in a law, if it can only intentionally refer to a type of phenomenon and if it may extensionally refer to general properties as well as to instances? The answer is not a complicated one. Consider again the example of the term "mass". Let $\varphi_{1}$ be the phenomenon type to which a law that involves the term "mass" refers and let $\varphi_{2}$ be the phenomenon type to which a prediction involving the same term refers. In this case, $\varphi_{2}$ be a sub-type of $\varphi_{1}$. For instance, let us say that $\varphi_{1}$ is the set of qualities of how objects resist to change their state of motion when they interact with other objects. The sub-type $\varphi_{2}$ may be a more restricted set of qualities, say, qualities of how medium-sized objects resist to change their state of motion.

As in (37), according to this last explanation, a term involved in two incommensurable theories can have the same reference for some domains of prediction. Predictions of these theories may have the same intensional reference as well as the same theoretically fixed, extensional reference, restricted to such domains.

\section{Conclusion}

According to the classical idea of semantic incommensurability, when two scientific theories are incommensurable, they are semantically incompatible regarding some relevant term, that is, they can be compared neither based on the sense nor based on the reference of that term. This does not imply that they cannot be compared at all. It only seems relevant to say that two theories are semantically incompatible if both are associated in some way with some field of phenomena. Although incommensurability implies relevant reference variation and the terms that ground semantic incompatibility cannot be compared based on the entities to which they correspond, it is possible to determine, between two incommensurable terms, which one serves better to describe the relevant phenomena with which they are associated. Thus, theory choice between incommensurable theories is possible and scientific progress is not threatened.

\section{Acknowledgements}

I would like to thank Rolando Rebolledo and Pamela Reyes for their helpful comments on a previous version of this work, which is result of a research financed by the Chilean Commission for Scientific and Technological Research (CONICYT / FONDECYT projects No. 1181414 and No 11180624).

\section{Bibliography}

Hoyningen-Huene, P. 1993. Reconstructing scientific revolutions: Thomas S. Kuhn's philosophy of science. Chicago: University of Chicago Press.

Recibido el 23 Jul 2018

Aceptado el 15 Mar 2019 